\title{
The Role of Umbilical Artery Doppler Velocimetry in Oligohydramnios ator Beyond 34 Weeks and Its Correlation withFetal Outcome
}

\author{
Manoj Mathur ${ }^{1}$,Saryu Gupta ${ }^{2}$, PreetKanwalSandhu Sibia ${ }^{3}$, Varinder Kaur ${ }^{4}$ \\ ${ }^{I}$ Associate Professor, Department of Radiodiagnosis and Imaging, Government Medical College and Rajindra \\ Hospital, Patiala (Punjab) India \\ ${ }^{2}$ Assistant Professor, Department of Radiodiagnosis and Imaging, Government Medical College and Rajindra \\ Hospital, Patiala (Punjab) India \\ ${ }^{3}$ Associate Professor, Department of Obstetrics \& Gynaecology, Government Medical College and Rajindra \\ Hospital, Patiala (Punjab) India \\ ${ }^{4}$ Junior Resident, Department of Radiodiagnosis and Imaging, Government Medical College and Rajindra \\ Hospital, Patiala (Punjab) India
}

\begin{abstract}
Amniotic fluid volume measurement forms an integral part of antenatal fetal monitoring in routine obstetric scans. Oligohydramnios has been identified as the leading cause of increased perinatal morbidity and mortality. Doppler ultrasound is extremely helpful in evaluating maternal and fetal hemodynamics in this scenario. In this study, we focus on the role of umbilical artery doppler velocimetry in oligohydramnios at or beyond 34 weeks and its correlation with fetal outcome.
\end{abstract}

Keywords: Color doppler, Oligohydramnios, Umbilical Artery

\section{Introduction}

Amniotic fluid plays a major role in the development of fetus. It provides a medium for fetal movement and growth. It also cushions the fetus against external injuries, maintains even temperature and prevents infection antenatally. During labour it helps in even distribution of the pressure of uterine contractions over the fetus, prevents cord compression and helps in cervical dilatation. Oligohydramnios was described as amniotic fluid index of $\leq 5 \mathrm{~cm}$ by Phelan et al[1]. It is associated with IUGR, FHR abnormalities, poor tolerance to the labour by the fetus, cord compression and low APGAR score with poor perinatal outcome. [2]. Umbilical artery Doppler assessment has been shown to reduce perinatal mortality and morbidity in high risk obstetric situations. [3] The fetal umbilical artery Doppler velocimetry evaluates the downstream impedance to the flow in the umbilical arteries. An abnormal umbilical artery Doppler velocimetry is an index of fetal peripheral vasoconstriction and associated with higher incidence of perinatal complications.

\section{Material And Methods}

This study consisted of analysis of usefulness of umbilical artery Doppler in pregnant women attending the antenatal clinic in department of obstetrics in RHP. This was a hospital based comparative analytical study done over a period of 22 months from December 2013 to October 2015. the study was divided into 3 groups. Group 1 consisted of 50 pregnant women with oligohydramnios AFI $<5 \mathrm{~cm}$ at or beyond 34 weeks of gestation with umbilical artery Doppler study. Group 2 consisted of 50 pregnant women with oligohydramnios AFI $<5 \mathrm{~cm}$ at or beyond 34 weeks of gestation. Control group consisted of 50 pregnant women with AFI- 5-20cms at or beyond 34 weeks of gestation. These groups were similar with regard to antepartum variables like maternal age, gravidity, gestational age and antepartum complications.

The inclusion criteria for this study consisted of Gestational age 34 weeks- 42 weeks, Singleton gestation with cephalic presentation, AFI $<5 \mathrm{~cm}$ and intact membranes. Patients with associated fetal genitourinary system malformations, gestational age less than 34 weeks, ruptured membranes, multiple gestation,intrauterine death, placenta previaandpolyhydramnios were excluded from the study. Informed consent was taken from all the patients participating in the study. For all the selected cases, thorough history was taken and complete examination was done. Previous records and reports were reviewed. Clinical evidence for oligohydramnios was looked for.For all women ultrasound examination was done and AFI was assessed by measuring the single deepest pocket or by using the four quadrant technique. Oligohydramnios was defined as AFI $<5 \mathrm{cms}$. Umbilical artery Doppler was done in oligohydramnios cases (50 cases) and comparison was done with cases having normal amniotic fluid index at or beyond 34 weeks of gestation. 


\section{Observations}

Maximum numbers of cases were found in the age group of 21-25 years in all study groups. Majority of cases belong to primigravida in all 3 groups. Mean gestational age for Group 1, 2, 3 was $38.26 \pm 1.64$ weeks, $38.72 \pm 1.51$ weeks and $38.74 \pm 1.96$ weeks respectively. Most cases of abnormal Doppler belong to 35 and 39 weeks of gestation. $80 \%$ of the cases were booked outside and were referred to our institution in view of pregnancy related complications like PET, severe PET, GDM, IUGR etc. Most cases belonged to middle socioeconomic status in all 3 groups.The common antenatal complication was pre-eclampsia accounting for $30 \%$ in group 1 and $16 \%$ in group 2. In cases with preeclampsia, $23.8 \%$ had abnormal umbilical artery Doppler findings and $32.22 \%$ had normal Doppler findings. In severe PET $9.5 \%$ had abnormal umbilical artery Doppler findings.

Group 1 had more cases in low AFI range as compared to group 2. The umbilical artery Doppler changes were more common in lower AFI range

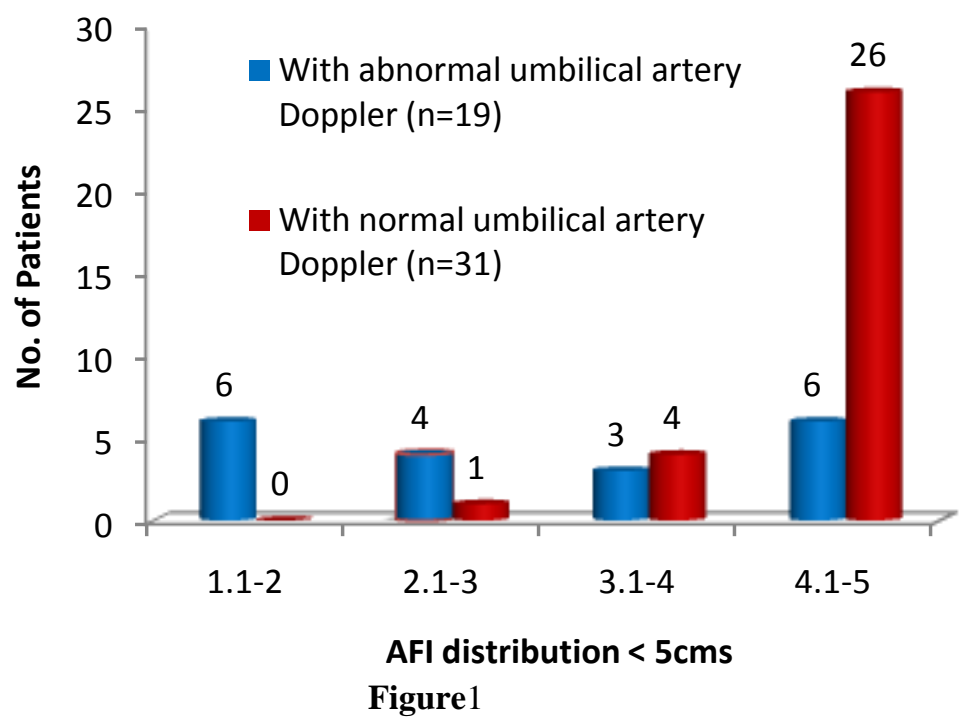

Incidence of non-reactive fetal heart tracing was more in study group 1 with $42 \%$ as compared to $30 \%$ in study group 2 and $20 \%$ on control group. FHR deceleration was more common in group 1 and group 2 as compared to control group. Incidence of thick meconium stained liquor was $40 \%$ in group 1 as compared to $6 \%$ in group 2 and $2 \%$ in group 3.

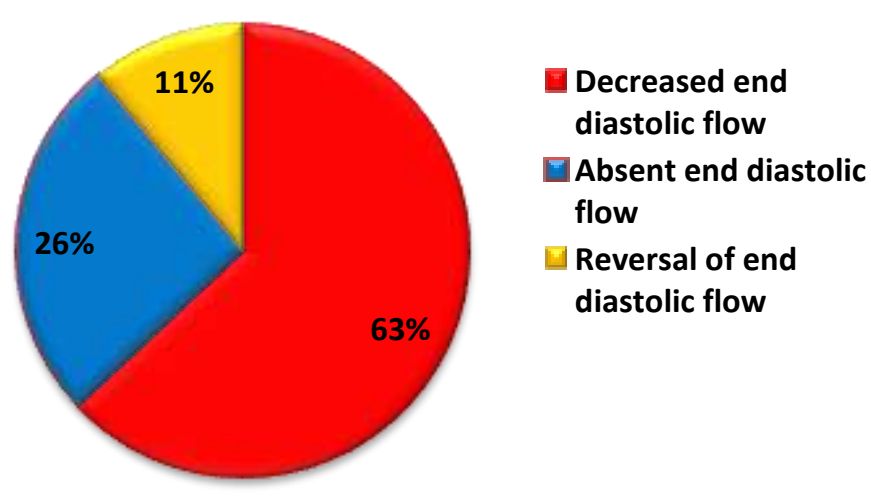

Figure 2

Labour induction in study group 1 was $26 \%$, study group 2 was $54 \%$ and in study group 3 was $40 \%$. Five cases $(26.3 \%$ ) with abnormal Doppler findings were taken for induction of labour. LSCS was frequent in study group 1 (72\%) compared to study group $2(54 \%)$ and control group (40\%). This was statistically significant (p- value 0.002098). Incidence of LSCS is $84.21 \%$ when umbilical artery Doppler studies are abnormal and $64.6 \%$ when the same were normal. LSCS for fetal distress was $55.6 \%$ in study group 1 as 
compared to $48 \%$ in study group 2. LSCS for fetal distress in group 1 with abnormal Doppler study was $52.6 \%$ and that for normal Doppler study was $32.25 \%$.

APGAR score at 1 minute was low in study group 1 as compared to group 2 and control group. Incidence of low APGAR score 1 min was $42 \%$ and $25.8 \%$ in abnormal and normal umbilical artery Doppler group respectively. The mean birth weight was less in oligohydramnios group.Statistical significance was observed ( $p$ value - 0.000). The number of NICU admissions were more common in study group 1 and group 2.Tachypnea and hyperbilirubinemia were the common indication for NICU admissions. This is statistically significant (p- value 0.0 01). NICU admissions was $84.2 \%$ and $42 \%$ in abnormal and normal umbilical artery Doppler group respectively.More number of babies in group $1(54 \%)$ were found to have IUGR compared to group $2(28 \%)$ and control group (10\%). Statistically significant association is observed in IUGR with p- value $<0.001$. Incidence of IUGR was $89.5 \%$ and $32.2 \%$ in abnormal and normal umbilical artery Doppler group respectively.Two cases with severe oligohydramnios and reversal of end diastolic flow, ended up in IUD in group 1. One neonate with oligohydramnios died due to meconium aspiration in group 2. (Figure 3,4 )

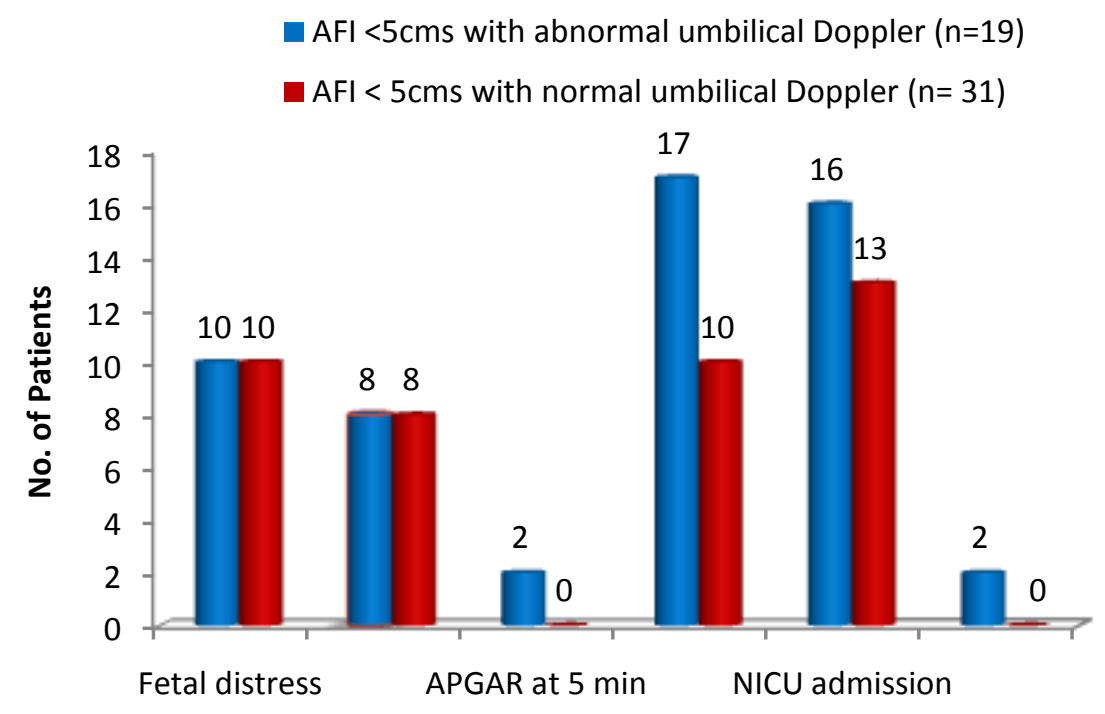

Perinatal outcome

Figure 3

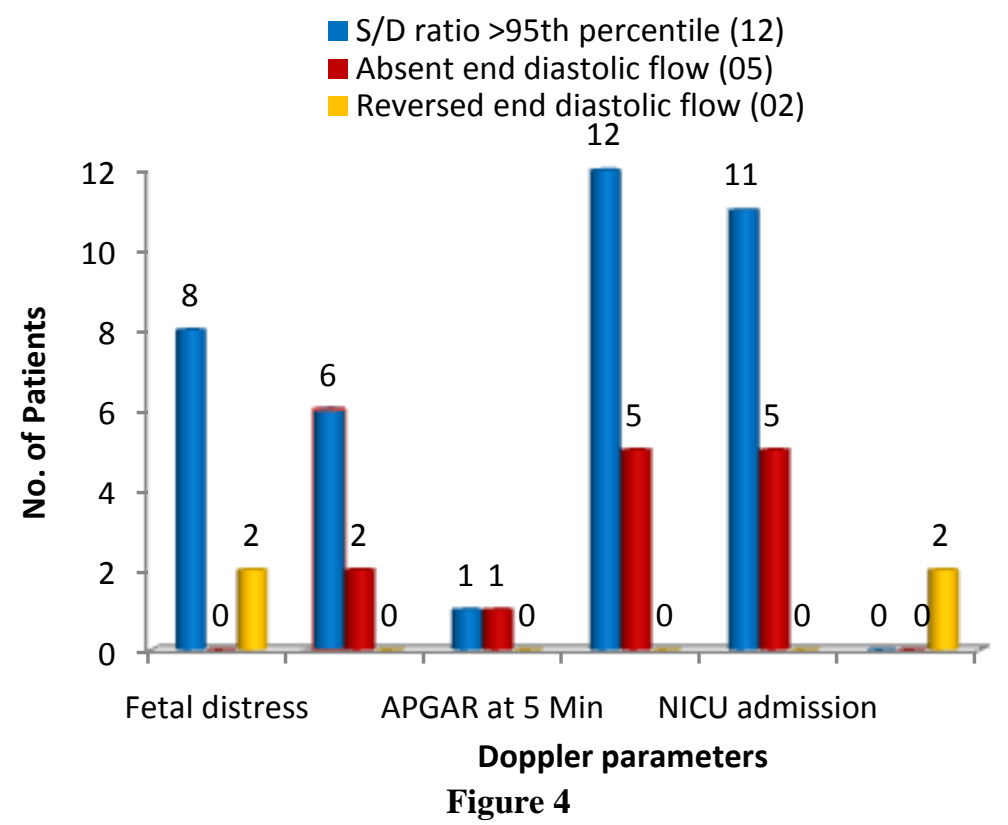




\section{Discussion}

The study was conducted on pregnant women between the gestational ages of 34 to 42 weeks admitted to Government medical college \& hospital, Patiala.The mean gestational age in the present study was 38.26 and 38.72 weeks, which is comparable with the mean gestational age of 37.5 weeks in a study conducted by Casey et al[4] (2000). Induction of labour was more common in oligohydramnios group which was also seen in the study by Casey et al[4] (2000). The occurrence of non-reactive NST was $42 \%$ in oligohydramnios and comparable to study conducted by Kumar et al[5] (1991) and Sriya et al[6] (2001). The ominous FHR pattern noted in 33\% in present study is comparable to study conducted by Casey et al[4] (2000) (48\%) and Sriya et al[6] (2001) (36.1\%). Thick meconium stained liquor was noted in $40 \%$ in study group in present study which is nearly similar to the studies conducted by Rutherford et al[7] (1987) and Sriya et al[6] (2001). The rate of LSCS for fetal distress in present study was $55.6 \%$ in grp $1 \& 48 \%$ in grp 2, while it was $51 \%$ \& $43 \%$ in studies by Casey et al[4] (2000) and Sriya et al[6] (2001).For oligohydramnios group (group 1 and group 2), the APGAR score at 1 minute was 54\%. This is comparable to the study conducted by Sriya et al[6] (2001). The incidence of low birth weight for group 1 was $52 \%$, which is comparable to the study conducted by Sriya R et al[6] (2001) and Casey et al[4] (2000). In the study conducted by Sriya et al[6] (2001) the incidence of NICU admissions was $88 \%$.In present study, out of 50 cases, $31(62 \%)$ had normal umbilical artery Doppler velocimetry and adverse perinatal outcome was $42 \%$ and $19(38 \%)$ had abnormal umbilical artery Doppler velocimetry and adverse perinatal outcome was $89.5 \%$. while in a study by carol et al[8], out of 81 cases of oligohydramnios, 50 patients $(61.7 \%)$ had normal S/D ratio, out of which 12 patients $(24 \%)$ were associated with identifiable perinatal morbidity where as in 31 patients (38.3\%) with abnormal Doppler findings, $74 \%$ had adverse perinatal outcome.

In present study, rate of induction of labour in cases with abnormal Doppler findings is $26.3 \%$ and that in cases with normal Doppler finding is $25.8 \% . \%$. In study performed by Arora et al[9] (2005), Induction of labour in their study was $29 \%$ in cases with normal Doppler findings and $17.1 \%$ in cases with abnormal Doppler findings. In present study, in cases with normal umbilical artery Doppler findings, 25.8\% had low APGAR and NICU admission was $42 \%$. In study performed by Arora et al[8] (2005), low APGAR score was $13.3 \%$ and NICU admission was 40\%. In cases with absent/reversal of diastolic flow, incidence of low APGAR score was $60 \%$ and NICU admission was $100 \%$ which is comparable to observation by Arora et al[9] (2005), 57\% and $100 \%$ respectively.

\section{Conclusion}

AFI $\leq 5 \mathrm{~cm}$ after 34 weeks of gestation is an indicator of poor perinatal outcome. Umbilical artery Doppler velocimetry in cases with oligohydramnios would help in identifying high risk cases for poor perinatal outcome. Hence in all patients with oligohydramnios umbilical artery doppler should be done to recognize the compromised fetus which will guide suitable interventional and management measures thereby reducing the perinatal morbidity and mortality.

\section{Declarations}

a. Funding: Nil

b. Conflict of interest: Nil

c. Ethical approval: Approved

\section{References}

[1]. Phelan JP, Smith CV, Broussard P, Small M. Amniotic fluid volume assessment with the four-quadrant technique at 36-42 weeks' gestation. J Reprod Med 1987; 32:540-2

[2]. Misra R. Hydramnios and Oligohydramnios. In: Ian Donald's Practical Obstetric Problems. (6th edn.) BI Publications Pvt Ltd. 2006;369-96.

[3]. Maulik D, Mundy D, Heitmann E et-al. Evidence-based approach to umbilical artery Doppler fetal surveillance in high-risk pregnancies: an update. ClinObstet Gynecol. 2010;53 (4): 869-78. doi:10.1097/GRF.0b013e3181fbb5f5 - Pubmed citation

[4]. Casey BM, McIntire DD, Bloom SL, Lucas MJ, Santos R, Twickler DM et al. Pregnancy outcome after diagnosis of oligohydramnios at or beyond 34 weeks of gestation. Am J ObstetGynecol 2000;182(4):902-12.

[5]. Kumar P, Iyer S, Ramkumar V. Amniotic fluid index: A new ultrasound assessment of amniotic fluid. J ObstetGynaecol India 1991;41(1):10-12.

[6]. Sriya R, Singhai S, Rajan M. Perinatal outcome in patients with amniotic fluid index $\leq 5 \mathrm{~cm}$. J ObstetGynaecol India 2001;51(5):98100 .

[7]. Rutherford SE, Jeffrey P, Phelan J, Smith CV, Jacobs N. The four-quadrant assessment of amniotic fluid volume: An adjunct to antepartum fetal heart rate testing. Obstet Gynecol. 1987;70(3 Pt 1):353-6.

[8]. Carroll BC and Bruner JP. Umbilical artery Doppler velocimetry in pregnancies complicated by oligohydramnios. J Reprod Med. 2000;45(7):562

[9]. Arora D, Desai SK, Sheth PN, KaniaP. Significance of umbilical velocimetry in perinatal outcome of growth restricted fetuses. J ObstetGynecol.India 2005;55(2):138. 